\title{
Ecosystem Services Linked to Water and Plant Diversity in the Igualata Paramo of Hualcanga Region
}

\section{Servicios Ecosistémicos Ligados al Agua y Diversidad Florística en el Páramo del Igualata Regional Hualcanga}

\section{S. Alvarez Cortez, R. Peña Murillo, and D. Román Robalino}

Memories II International Congress Forests and Agroforestry for the 21st Century

Corresponding Author:

S. Alvarez Cortez

Published: 21 January 2021

Production and Hosting by Knowledge E

(c) S. Alvarez Cortez et al. This article is distributed under the terms of the Creative Commons Attribution License, which permits unrestricted use and redistribution provided that the original author and source are credited.
Facultad de Recursos Naturales, Escuela Superior Politécnica de Chimborazo, Riobamba, Ecuador

\section{Abstract}

The present investigation proposes to study the ecosystem services linked to water and floristic diversity in the Igualata paramo of the Hualcanga region; 5 sampling plots of $25 \mathrm{~m}^{2}$ were installed, with 4 subplots of $1 \mathrm{~m}^{2}$. 30 samples of terrestrial vascular plants were obtained, identifying 17 botanical families, 26 genera, and 27 species, and 1 lichen and 1 moss of unidentified family and genus. It was determined that the species that stands out with the highest importance value index was Lachemilia orgiculata with $44.06 \%$, because the species was found in 4 of the 5 study plots. In second place we have Calamagrostis intermedia species with $8.21 \%$, while the species Vicea $s p$. was the species with the lowest importance value index of $0.50 \%$, being the species with the lowest number of individuals. The Rosaceae family presented the highest index with $39.61 \%$. The Poaceae and Asteraceae families also present dominance in this ecosystem with values of $9.63 \%$ and $5.79 \%$, respectively. According to the Shannon index, plots P2 and P4 were those with high diversity, which corroborates with what was obtained in the Simpson index; the Sorensen index showed us that plots P4 and P5 are very similar, plots P1 and P3 are fairly similar, and the remaining plots are dissimilar, which indicated that vegetation can be found in certain areas of the investigation. The water flow supplying the resident families of the region was found to be $8.12 \mathrm{~L} / \mathrm{s}$, and its ecological flow is of $0.83 \mathrm{~L} / \mathrm{s}$, having a flow that satisfies the needs of the inhabitants.

Keywords: caudal, ecological flow, ecosystem paramo, ecosystem services, floristic diverity, indices of diversity.

\section{Resumen}

La presente investigación propone: estudiar los servicios ecosistémicos ligados al agua y diversidad florística en el páramo del Igualata regional Hualcanga; se instalaron cinco parcelas de muestreo de $25 \mathrm{~m}^{2}$ con 4 sub parcelas de $1 \mathrm{~m}^{2}$, se obtuvo 30 muestras de plantas vasculares terrestres, identificando 17 familias botánicas, 26 géneros y 27 especies, además se colectó 1 liquen y 1 musgo de familia, género y especie no identificado. Se determinó que la especie que sobresale con mayor índice de valor de importancia fue Lachemilia orgiculata con un 44,06\%, esto se debe a que la especie se encontraba en 4 de las 5 parcelas de estudio, en segundo lugar, tenemos a Calamagrostis intermedia especie con 8,21\%, mientras que la especie Vicea sp. fue la que menor índice de valor de importancia presentó con un 0,50\% esto se debe a que 
fue la especie con menor cantidad de individuos. La familia Rosaceae presentó el mayor índice con $39,61 \%$, las familias Poaceae, y Asteraceae presentan una dominancia en este ecosistema con valores de 9,63\% 5,79\%, respectivamente. De acuerdo al índice de Shannon las parcelas 2 y 4 son las que poseen diversidad alta que se corrobora con lo obtenido en el índice de Simpson; en el índice de Sorensen nos muestra que las parcelas P4 vs P5 son muy similares las parcelas P1 y P3 son medianamente similares y las parcelas restantes son disimiles lo que indica que la vegetación se puede encontrar en ciertas zonas de la investigación. El caudal que posee la Regional es de $8,12 \mathrm{~L} / \mathrm{s}$, abasteciendo a las familias y su caudal ecológico es de $0,83 \mathrm{~L} / \mathrm{s}$ teniendo un caudal que satisface las necesidades de los pobladores.

Palabras Clave: caudal, caudal ecológico, diversidad florística, ecosistema páramo, índices de diversidad, servicios ecosistémicos.

\section{Introducción}

Los páramos son ecosistemas semi - húmedos y fríos que en el Ecuador se encuentran formando un corredor casi intacto sobre la Cordillera de los Andes, por encima del límite superior actual o potencial de bosque [1]. La recarga hídrica es sin duda la cualidad más importante de los páramos, esta importancia se debe a un balance hídrico positivo, también se debe a la estructura de la vegetación que capta el agua, la conduce al suelo y lo protege contra erosión y disecación. Se puede decir que cada metro cuadrado de páramo 'produce' 1 L de agua por día [2]. La diversidad del páramo no está reflejada solo en su flora, fauna y paisaje, sino también en sus habitantes. La diversidad cultural y étnica hacen que la alta montaña, aparte de la Amazonía, sea el único lugar donde todavía se hallan rasgos del Ecuador nativo, indígena. La mayor población indígena Quichua hablante vive en los páramos, practica su agricultura con algunas prácticas muy tradicionales, habla su idioma, tiene su cultura y vestimenta y está en un continuo proceso de cambio y adaptación, lo que quiere decir que es una cultura diversa y viva [3]. El desconocimiento de la conservación del páramo provoca que los comuneros destruyan estos ecosistemas afectando los suelo que serán destinados para actividades económicas como los cultivos agrícolas, la ganadería, las plantaciones forestales, la minería, el manejo inadecuado del agua y la expansión territorial amenazando severamente el ecosistema páramo. Los páramos alto andinos poseen una variedad de ecosistemas y especies, mismos que han permitido desarrollar una amplia diversidad biológica, constituyen un capital natural que es necesario para conservar y para garantizar la permanencia de servicios como la regulación del clima, fijación de carbono, fertilidad del suelo, polinización, provisión de agua limpia, reducción de desastres naturales, recreación, valores estéticos, espirituales, entre otros. Los servicios brindados por los ecosistemas tienen impactos positivos en el desarrollo de la sociedad, por lo que, el mantenimiento en buen estado y conservación de las áreas protegidas representa una estrategia eficiente para mantener las funciones y servicios que sus complejos ecosistémicos brindan y los beneficios que reportan a las comunidades locales. 


\section{Materiales y Métodos}

\section{1. Área de estudio}

El Páramo del Igualata se encuentra ubicado en el Cantón Quero - Provincia de Tungurahua formando parte de la Regional Hualcanga, la misma que se sitúa en la cuenca del río Pastaza, subcuenca de los ríos Ambato y Chambo, el flujo principal forma el río Ambato que se origina en los páramos del Frente Sur Occidental. La regional obtiene el agua de vertientes del sector La Ciénega, Isco Y Gualimbo a alturas que van dese los 3900 hasta los 4200 m.s.n.m. En el área de estudio podemos distinguir 2 zonas de vida: Arbustal siempre verde y Herbazal del Páramo que se encuentra de 3000-3700 m.s.n.m. Y Herbazal húmedo montano alto superior del Páramo que se encuentra de 3700-4200 m.s.n.m. [4].

Se realizó 20 sub-parcelas de $1 \mathrm{~m}^{2}$ (Tabla 1) y 3 zonas de aforo (Figura 1).

\section{Table 1}

Ubicación de las sub-parcelas de muestreo en la regional.

\begin{tabular}{|c|c|c|c|}
\hline Punto & $x$ & $\mathbf{Y}$ & Altura \\
\hline P 1.1 & 763990 & 9834245 & $3828 \mathrm{~m}$ \\
\hline P 1.2 & 763994 & 9834227 & 3975 m \\
\hline P 1.3 & 764015 & 9834238 & 3970 m \\
\hline P 1.4 & 764019 & 9834251 & $3980 \mathrm{~m}$ \\
\hline P 2.1 & 764207 & 9834230 & 3918 m \\
\hline P 2.2 & 764226 & 9834238 & 3919 m \\
\hline P2 3 & 764234 & 9834229 & $3314 \mathrm{~m}$ \\
\hline P 2.4 & 764226 & 9834219 & 3910 m \\
\hline P 3.1 & 764849 & 9834245 & $3835 \mathrm{~m}$ \\
\hline P 3.2 & 764853 & 9834238 & 3831 m \\
\hline P 3.3 & 764839 & 9834229 & $3835 \mathrm{~m}$ \\
\hline P 3.4 & 764835 & 9834238 & 3831 m \\
\hline P 4.1 & 765097 & 9834357 & 3817 m \\
\hline P 4.2 & 765090 & 9834366 & 3818 m \\
\hline P 4.3 & 765111 & 9834367 & 3818 m \\
\hline P 4.4 & 764835 & 9834355 & 3812 m \\
\hline P 5.1 & 765177 & 9834400 & $3804 \mathrm{~m}$ \\
\hline P 5.2 & 765190 & 9834399 & 3807 m \\
\hline P 5.3 & 765195 & 9834404 & 3807 m \\
\hline P 5.4 & 765183 & 9834416 & $3809 \mathrm{~m}$ \\
\hline
\end{tabular}




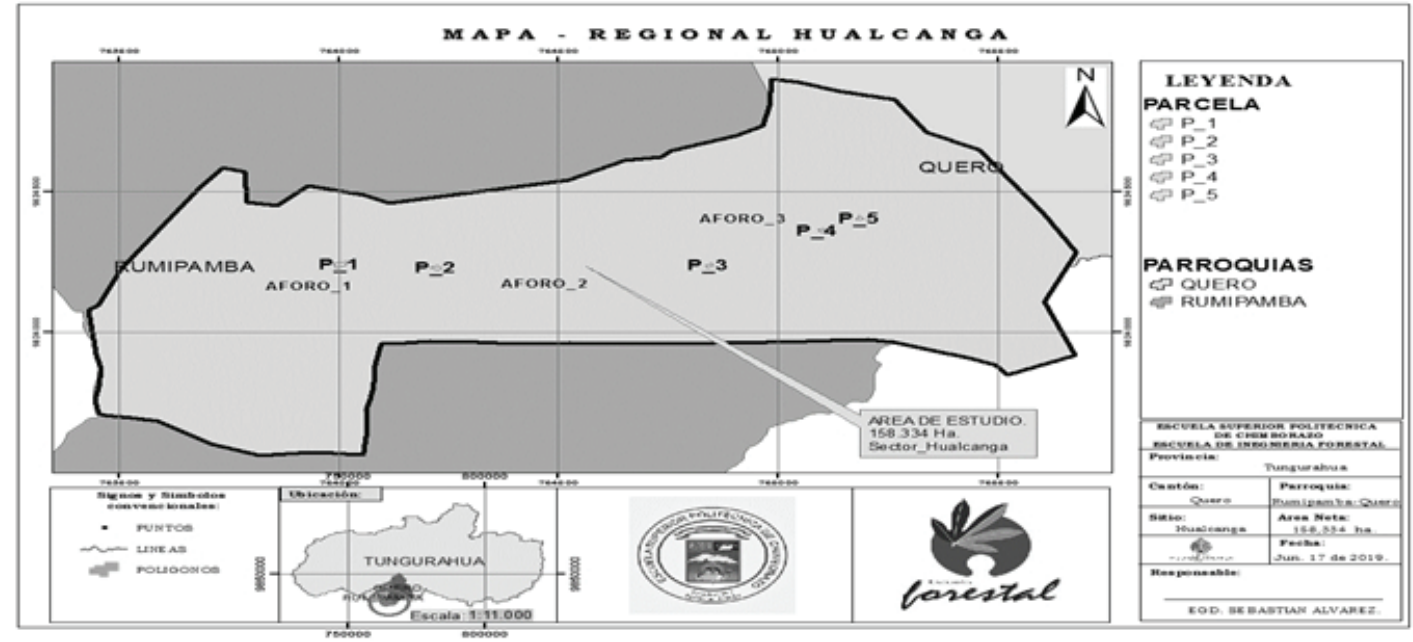

Figure 1

Ubicación de las parcelas de muestreo y zonas de aforo en la Regional San José de Chazo-Santa Fe de Galán.

\subsection{Diseño de muestreo}

\subsubsection{Diversidad florística}

Para la instalación de parcelas se tomó en cuenta la metodología propuesta por [5] citado por [6] para el Proyecto Gloria tomando en cuenta algunas modificaciones para adaptar a los páramos andinos. Se instalaron 5 parcelas de muestreo de $5 \times 5 \mathrm{~m}$, ubicadas a $300 \mathrm{~m}$ de distancia y en distintos lugares tomando en cuenta la pendiente, accesibilidad y el estado de cada sitio. Cada parcela de muestreo fue dividida en subparcelas de 1×1 m, las observaciones y recolección de vegetación se llevó a cabo únicamente en las sub-parcelas de las esquinas ya que las otras se alteraron con el pisoteo mientras se realizaba la recolección de las muestras, obteniéndose el registro de 20 sub-parcelas de $1 \times 1 \mathrm{~m}$. Los sub-cuadrantes de $1 \mathrm{~m} \times 1 \mathrm{~m}$, se sub-dividieron en celdillas de 0,1×0,1 m, para lo cual se utilizó un armazón de madera con un enrejado de hilos que delimitaron un total de 100 celdillas.

Las especies vegetales que se recolectaron en la zona de estudio fueron herborizadas en el mismo lugar, las especies fueron secadas manualmente bajo sombra, se cambió de papel periódicamente para evitar la putrefacción de las muestras o que estas se llenen de hongos. Dentro de las parcelas se registró número de individuos, frecuencia y cobertura de cada especie, con el fin de obtener datos cuantitativos de la vegetación, las especies que no se pudieron identificar en el campo fueron registradas con códigos para su posterior identificación en el herbario de la ESPOCH.

\subsubsection{Caudal}

En la zona de estudio se identificó tres zonas de aforo: Zona 1 (vertiente), Zona 2 (Receptor 1) y Zona 3 (Tanque de distribución), se tomó las coordenadas UTM de cada 
zona. Se utilizó el método volumétrico, el cual consistió en llenar un balde de $10 \mathrm{~L}$ y tomar el tiempo que este tarda en llenarse. El método se efectuó con 3 repeticiones.

\subsection{Análisis de datos}

\subsubsection{Diversidad florística}

Con la información de las especies vegetales se obtuvo: Índice de valor de importancia (IVI) por especie y familia, índice de Shannon-Weaver, índice de Simpson e índice de Sorensen. Estos índices corresponden a una medida de la heterogeneidad de una comunidad en función de la riqueza y la abundancia de las especies. La diversidad permite distinguir entre dos comunidades con idéntica riqueza y composición florística, en la cual las especies difieren en cuanto a su abundancia relativa [7].

Para tener idea de (número de individuos de una especie con relación al total de individuos de la población) [8] citado por [9]. La densidad relativa se refiere al porcentaje con el que cada especie contribuye al conjunto de la comunidad [10] citado por [9].

$$
\mathrm{Dr}=\frac{\text { total de individuos especie } \mathrm{A}}{\text { total de individuos, todas las especies }} \times 100
$$

Frecuencia relativa (FR).

$$
\text { Frecuencia Relativa }=\frac{\text { valor de frecuencia de la especie } A}{\text { valor total de frecuencia, todas las especies }} \times 100
$$

Dominancia relativa.

$$
\text { Dominancia relativa }=\frac{\text { área cobertura sp } \mathrm{A}}{\text { área de cobertura de todas las } \mathrm{sp}} \times 100
$$

El Índice de diversidad de Shannon es un índice basado en la equidad, expresa la uniformidad de los valores de importancia a través de todas las especies de la muestra [11]. Los valores se interpretaron de acuerdo a la Tabla 2.

$$
H^{\prime}=-\sum_{i=1}^{S} p i\left(\text { In } p_{i}\right),
$$

dónde $H^{\prime}$ es el Índice de Shannon; S es el número de especies; $p_{i}$ es la proporción del número total de individuos que constituyen la especie.

El Índice de diversidad de Simpson está basado en la dominancia, son parámetros inversos al concepto de uniformidad o equidad de la comunidad. Tomando en cuenta la representatividad de las especies con mayor valor de importancia sin evaluar la contribución del resto de las especies [11]. Los valores se interpretaron de acuerdo a la Tabla 2.

$$
I S D=1-\sum(p i)^{2},
$$

dónde ISD es el Índice de Simpson; pi es la proporción del número total de individuos que constituyen la especie. 


\section{Table 2}

Interpretación del Índice de Shannon y Simpson.

Valores
$0,00-0,35$
$0,36-0,75$
$0,76-1,00$

Fuente: Referencias [12, 13].

\section{Interpretación \\ Diversidad baja \\ Diversidad mediana \\ Diversidad alta}

El Índice de diversidad de Sorensen no considera la abundancia relativa de las especies. Es mucho más útil cuando el principal interés es la determinación de la presencia o ausencia de las especies [10] citado por [9]. Los valores se interpretaron de acuerdo a la Tabla 3.

$$
I s s=\frac{2 C}{A+B} \times 100,
$$

dónde Iss es el Índice de Sorensen; $A$ es el número de especies en el sitio 1; $B$ es el número de especies en el sitio 2; $C$ es el número de especies similares presentes en ambos sitios $A$ y $B$.

\section{Table 3}

Interpretación del Índice de Sorensen.

Valores
$0,00-0,35$
$0,36-0,70$
$0,71-1,00$

\section{Interpretación}

Disimiles

Medianamente similares

Muy similares

Fuente: Referencias $[9,12]$.

\subsubsection{Caudal}

El caudal se entiende que es el volumen de agua que traspasa una superficie en una unidad de tiempo [14].

Se aplicó la fórmula propuesta por la Guía de orientación en Saneamiento Básico (S.F.):

$$
Q=\frac{V}{T},
$$

dónde: Q: Caudal; V: L (litros); T: s (segundos). 


\section{Resultados y Discusión}

\subsection{Riqueza florística}

Se colectó 30 muestras de plantas vasculares terrestres, identificando 17 familias botánicas, 26 géneros, 27 especies y 15.229 individuos, las cuales han sido identificadas en el Herbario de la ESPOCH. Además, también se colectó un liquen y un musgo de familia, género y especie no identificado (Tabla 4).

\subsubsection{Composición florística de las 5 parcelas de muestreo}

Caranqui et al. [15], menciona que la presencia de un mayor o menor número de especies está influenciada tal vez por el estado de conservación de los páramos o por el grado de intervención de estos tipos de vegetación. Así en los resultados obtenidos se distingue que la Parcela 5 es la que mayor riqueza posee con 11 familias, 12 géneros y 12 especies, seguida de las Parcelas 1 y 4 con 8 familias, 10 géneros y 10 especies, como se indica en el cuadro 8 y en el gráfico 2. Por otro lado, la Parcela 2 encontramos 6 familias, 6 géneros y 6 especies siendo la parcela con menos diversidad en comparación con la Parcela 3, esto podría deberse a la zona de estudio se encuentra en recuperación ya que anteriormente este sitio estaba en proceso de degradación por actividades antrópicas.

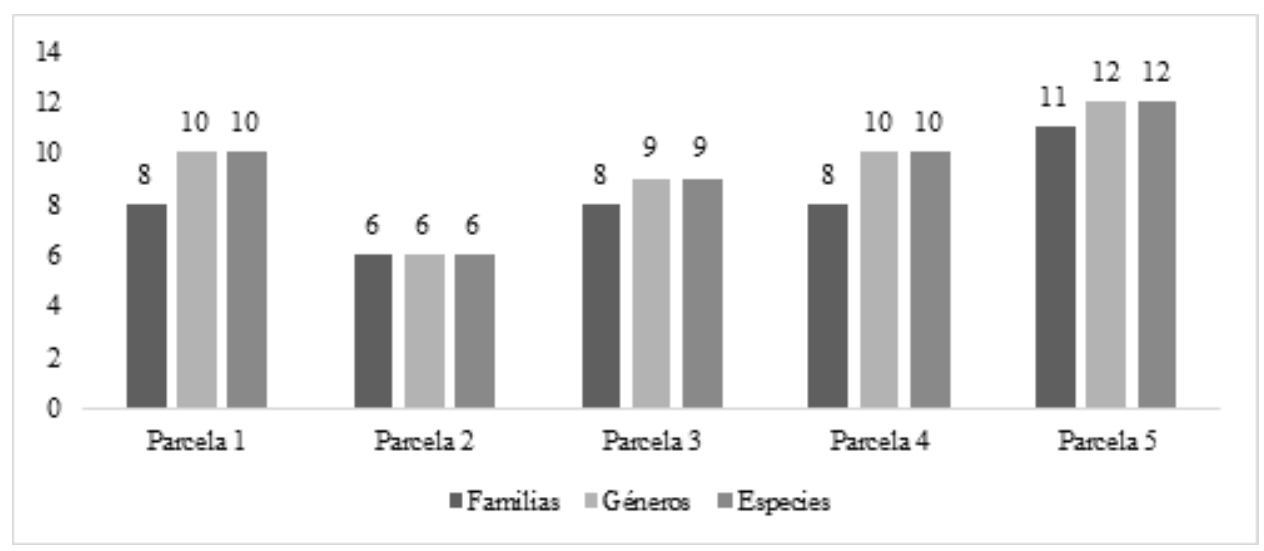

Figure 2

Composición florística de las 5 parcelas.

\section{2. Índice de valor de importancia por especie}

Según el Figura 3 la especie que sobresale con mayor índice de valor de importancia en la Regional, fue Lachemilia orgiculata con un $44,06 \%$, esto se debe a que la especie se encontraba en 4 de las 5 parcelas de estudio, según Caranqui [16] este tipo de especies puede llegar a cubrir hasta el $100 \%$ de la superficie y son indicadores de un existió pastoreo en la zona, estas almohadillas protegen los suelos de la erosión acumulando agua y protegiendo los órganos jóvenes de las demás especies en segundo lugar 


\section{Table 4}

Vegetación registrada.

\begin{tabular}{|c|c|c|c|c|}
\hline Familia & Nombre & $\mathrm{Ge}$ & Sp & Individuos \\
\hline Apiaceae & $\begin{array}{l}\text { Daucus montanus Humb et } \\
\text { Bonpl. ex spreng }\end{array}$ & 2 & 2 & 23 \\
\hline Apiaceae & Eryngium humile Cav & & & 53 \\
\hline Asteraceae & Hypochaeris sessiliflora Kunth & 3 & 4 & 23 \\
\hline Asteraceae & Bidens andicola Kunth & & & 64 \\
\hline Asteraceae & Metameridis & & & 2 \\
\hline Asteraceae & $\begin{array}{l}\text { Baccharis caespitosa (Lam.) } \\
\text { Pers }\end{array}$ & & & 6 \\
\hline Blechanaceae & Blechnum & 1 & 1 & 60 \\
\hline Caprifoliaceae & Valeriana microphylla & 1 & 1 & 106 \\
\hline Dryopteridaceae & Elaphoglossium & 2 & 2 & 900 \\
\hline Dyopteridaceae & Polystichum orbivulatum & & & 18 \\
\hline Ericaceae & Disterigma empetrifolium & 2 & 2 & 18 \\
\hline Ericaceae & Vaccinium floribundum Kunth & & & 49 \\
\hline Fabacea & Vicea & 1 & 1 & 4 \\
\hline Gentianaceae & Gentianella & 2 & 2 & 7 \\
\hline Gentianaceae & Gentiana sedifolia & & & 10 \\
\hline Grosularaceae & Ribes andicola & 1 & 1 & 10 \\
\hline Hyperocaceae & Hypericum sp. & 1 & 1 & 2 \\
\hline Indeterminada & Liquen & & & 76 \\
\hline Indeterminada & Musgo & & & 166 \\
\hline Leguminosa & Geranium laxicaule & 1 & 1 & 585 \\
\hline Plantaginaceae & Plantago australis & 1 & 1 & 150 \\
\hline Poaceae & $\begin{array}{l}\text { Calamagrostis intermedia (J. } \\
\text { Presl) Steud. }\end{array}$ & 3 & 3 & 457 \\
\hline Poaceae & Holcus lanatus $L$. & & & 57 \\
\hline Poaceae & Festuca cf & & & 3 \\
\hline Polygonaceae & Rumex acetosella $L$ & 1 & 1 & 38 \\
\hline Pteridaceae & Pellacea termifolia & 1 & 1 & 62 \\
\hline Ranunculaceae & Ranunculus praemersus & 1 & 1 & 14 \\
\hline Rosacea & Polilepys racemosa & 2 & 2 & 12 \\
\hline Rosacea & Lachemilia orgiculata & & & 12254 \\
\hline 17 & & 26 & 27 & 15229 \\
\hline
\end{tabular}

tenemos a Calamagrostis intermedia (J. Presl) Steud.como con 8,21\% lo cual concuerda con el estudio de Caranqui et al. [15] además esta especie es propia de los páramos de pajonal según la clasificación de Proyecto Páramo 1999. Mientras que la especie Vicea fue la que menor índice de valor de importancia presentó con un 0,50\% esto se debe a que fue la especie con menor cantidad de individuos, seguida por otras especies registradas en este ecosistema. 


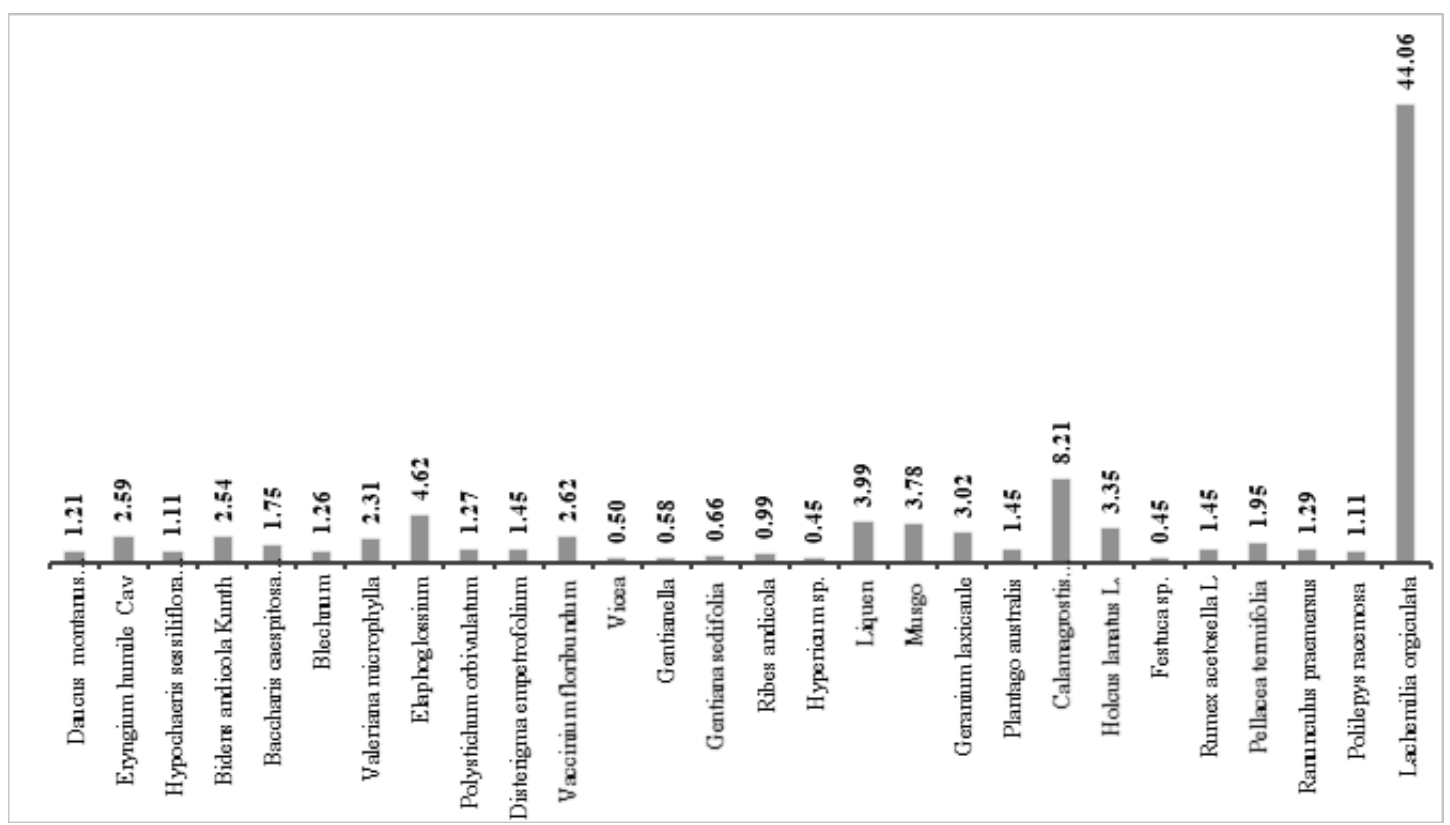

Figure 3

Índice de valor de importancia por especie.

\section{3. Índice de valor de importancia por familia}

Según el Figura 4 la familia que sobresale con mayor índice de valor de importancia en la Regional, fue la familia Rosaceae con un $39,61 \%$, esto se debe a que la especie se encontraba en 4 de las 5 parcelas de estudio, las familias Poaceae, y Asteraceae también presentan una dominancia en este ecosistema con valores de $9,63 \%$ y $5,79 \%$, respectivamente lo que concuerda con la aseveración de Bayas [13] y Hofstede et al. [17], quienes mencionan que las familias más representativas en los páramos del Ecuador son Asterácea, Poaceae, Rosácea. También afirman que en los páramos de Ecuador las familias Asteraceae, Poaceae y Rosacea son las familias más ricas en géneros y especies, seguidas por las familias (Ericaceae, Orchidaceae, Cyperaceae, Gentianaceae, Scrophulariaceae, Apiaceae),

\section{4. Índice de Shannon e Îndice de Simpson}

Como se muestra en la Tabla 5, el índice de diversidad de Shannon en la parcela No. 03 posee una diversidad baja, mientras que las parcelas No. 01 y 05 tienen una diversidad media, la parcela No. 04 fue la parcela que mayor índice de diversidad presentó, estos resultados obtenidos concuerdan con la Tabla 4 mencionada por Ordoñez et al. [12] en donde indica que mientras mayor sea el número referencial mayor riqueza tendrá dentro de la zona de estudio (Tabla 5).

En la Tabla 6 nos muestra el índice de diversidad de Simpson en donde las parcelas No. 01, 03, 05 poseen diversidad baja, mientras que las parcelas No. 02, 04 poseen diversidad media, en ninguna de las 5 parcelas de muestreo de la zona de estudio 


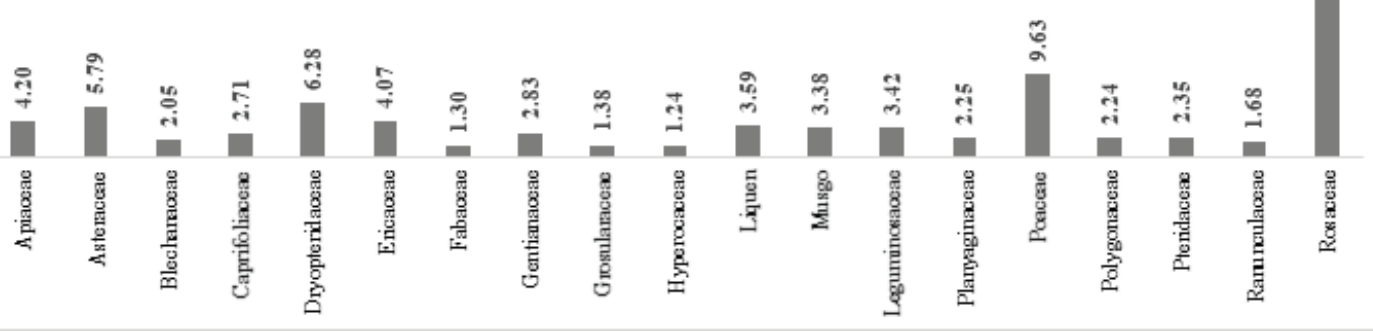

\section{Figure 4}

Índice de valor de importancia por familia.

registró diversidad alta. La biodiversidad de la zona de estudio se sitúa en diversidad baja de acuerdo a la interpretación por Ordoñez et al. [12] además estos resultados se corroboran con lo observado en la zona de estudio.

\section{Table 5}

Índice de Shannon-Weaver por parcela.

\begin{tabular}{l|l|l|l} 
No. parcela & Valor calculado & Valor referencial & Interpretación \\
\hline P 1 & 0,63 & $0,36-0,75$ & Diversidad media \\
\hline P 2 & 0,98 & $0,00-0,35$ & Diversidad baja \\
\hline P 3 & 0,32 & $0,76-1,00$ & Diversidad alta \\
P 4 & 1,12 & $0,76-1,00$ & Diversidad alta \\
P 5 & 0,52 & $0,36-0,75$ & Diversidad media
\end{tabular}

\section{Table 6}

Índice de Simpson por parcela.

No. parcela
P 1
P 2
P 3
P 4
P 5

Valor calculado
0,30
0,51
0,11
0,45
0,19

Valor referencial
$0,00-0,35$
$0,00-0,35$
$0,36-0,75$
$0,36-0,75$
$0,00-0,35$

Interpretación
Diversidad baja
Diversidad baja
Diversidad media
Diversidad media
Diversidad baja

\section{5. Índice de Sorensen}

Como se observa en la Tabla 7 las parcelas de muestreo indican que en su mayoría son disimiles, es decir, que hay especies vegetales que se pueden encontrar en ciertas 
zonas del área de estudio, la parcela 4 vs la parcela 5 muestran ser muy similares, la parcela 1 vs la parcela 3 presentan similitud media la interpretación se realizó de acuerdo a la Tabla 5 mencionada Ordoñez et al. [12].

\section{Table 7}

Índice de Sorensen (similitud entre parcelas).

No. de parcela
P 1 vs P 2
P 1 vs P 3
P 1 vs P 4
P 1 vs P 5
P 2 vs P 3
P 2 vs P 4
P 2 vs P 5
P 3 vs P 4
P 3 vs P 5
P 4 vs P 5

Valor calculado
0,13
0,53
0,20
0,18
0,13
0,25
0,22
0,32
0,29
0,82

Valor referencial
$0,36-0,70$
$0,36-0,70$
$0,36-0,70$
$0,36-0,70$
$0,36-0,70$
$0,36-0,70$
$0,36-0,70$
$0,36-0,70$
$0,36-0,70$
$0,36-0,70$

Interpretación
Disímiles
Medianamente similares
Disímiles
Disímiles
Disímiles
Disímiles
Disímiles
Disímiles
Disímiles
Muy similares

\subsection{Caudal medio mensual}

Según la Tabla 8 se observa una variación del caudal mensual durante el periodo de registro febrero - Mayo, presentándose para la zona 1 (vertiente) 7,15 L/s; 7,52 L/s; 7,09 L/s; 7,05 L/s; zona 2 (receptor 1) 6,77 L/s; 7,26 L/s; 6,71 L/s; 6,62 L/s y zona 3 (tanque de distribución) 10,10 L/s; $10,20 \mathrm{~L} / \mathrm{s} ; 10,51 \mathrm{~L} / \mathrm{s} ; 10,47 \mathrm{~L} / \mathrm{s}$, respectivamente, obteniéndose un caudal promedio total de 8,12 L/s. Los páramos son considerados como grandes humedales, por sus condiciones de suelo y vegetación además de ser buenos retenedores de agua [17].

Table 8

Registro de caudal mensual y medio mensual.

\begin{tabular}{|c|c|c|c|c|c|c|}
\hline \multirow[t]{2}{*}{ Zona } & \multicolumn{4}{|c|}{ Registro mensual del caudal (L/s) } & \multirow{2}{*}{$\begin{array}{l}\text { Caudal } \\
\text { promedio/ } \\
\text { zona }\end{array}$} & \multirow{2}{*}{$\begin{array}{l}\text { Caudal } \\
\text { promedio } \\
\text { total (L/s) }\end{array}$} \\
\hline & Febrero & Marzo & Abril & Mayo & & \\
\hline Vertiente & 7,15 & 7,52 & 7,09 & 7,051 & 7,20 & 8,12 \\
\hline 1er Receptor & 6,77 & 7,26 & 6,71 & 6,623 & 6,84 & \\
\hline Tanque de distribución & 10,10 & 10,20 & 10,51 & 10,471 & 10,32 & \\
\hline Caudal Promedio/ mes (L/s) & 8,01 & 8,33 & 8,10 & 8,05 & & \\
\hline
\end{tabular}




\subsection{Relación caudal - Precipitación}

Según la Figura 5 observamos la relación existente entre precipitaciones y el caudal siendo el mes de marzo que registró mayor caudal con 8,33 L/s con una precipitación de $80,60 \mathrm{~mm}$ y en el mes de febrero registro el menor caudal con $8,01 \mathrm{~L} / \mathrm{s}$ con una precipitación $30,70 \mathrm{~mm}$, además se observa que la curva de tendencia presenta una característica polinómica de segundo orden cuya correlación es de 0,89 existiendo una relación positiva, es decir que, a mayor precipitación, mayor será el caudal.

Según el INAMHI [18], en el Ecuador existe dos temporadas una seca que va desde junio - noviembre y una húmeda diciembre - mayo, los datos registrados fueron tomados en la época húmeda además estas relaciones de precipitación y caudal coinciden con lo mencionado por Estrella et al. [19].

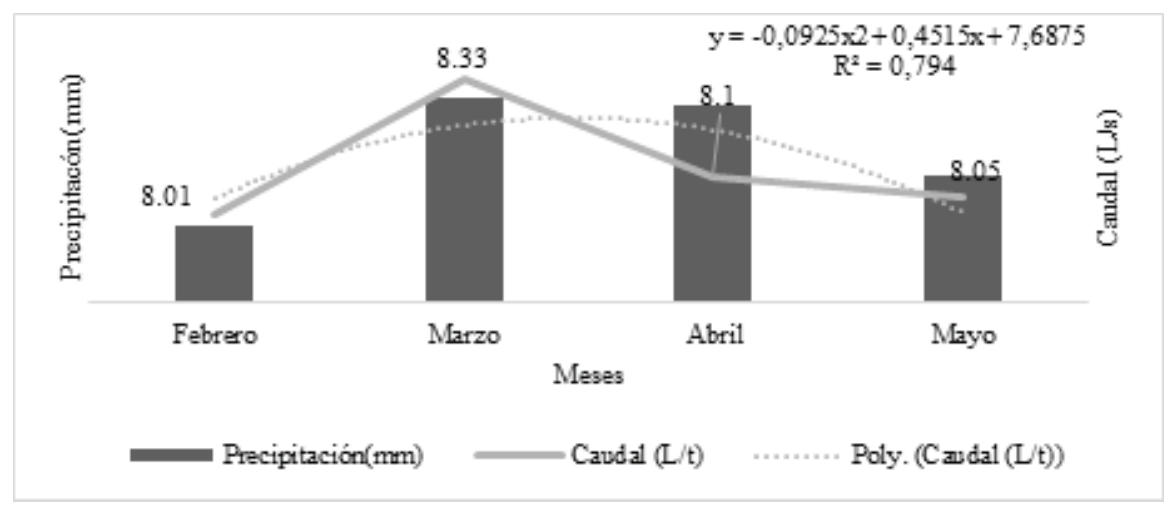

\section{Figure 5}

Relación precipitación caudal en los meses de febrero a mayo.

\subsection{Caudal promedio por zona}

Como se observa en la Figura 6 el mayor caudal promedio por zona de aforo es la zona 3 (tanque de distribución) con 10,32 L/s ya que aquí existen afluentes tributarios que producen un aumento de caudal, indicando también que la zona 1 (vertiente) posee 7,20 $\mathrm{L} / \mathrm{s}$, lo cual concuerda con Bob [20] donde menciona que cada punto de aforo al menos tenga $0,125 \mathrm{~L} / \mathrm{s}$, además la curva de tendencia presenta una característica polinómica de segundo orden cuya correlación es de 0,89 evidenciando una correlación en los datos registrados.

\subsection{Caudal ecológico}

Como se observa en la Figura 7 el caudal ecológico, en el mes de marzo fue de 0,83 L/s siendo el mes con mayor caudal, los meses restantes mantiene un equilibrio el mismo que de acuerdo a Bozeman [21] puede ser considerado como caudal 'excelente' ya que poseen un promedio de $0,8 \mathrm{~L} / \mathrm{s}$ satisfaciendo las necesidades de los beneficiarios de la Regional. 


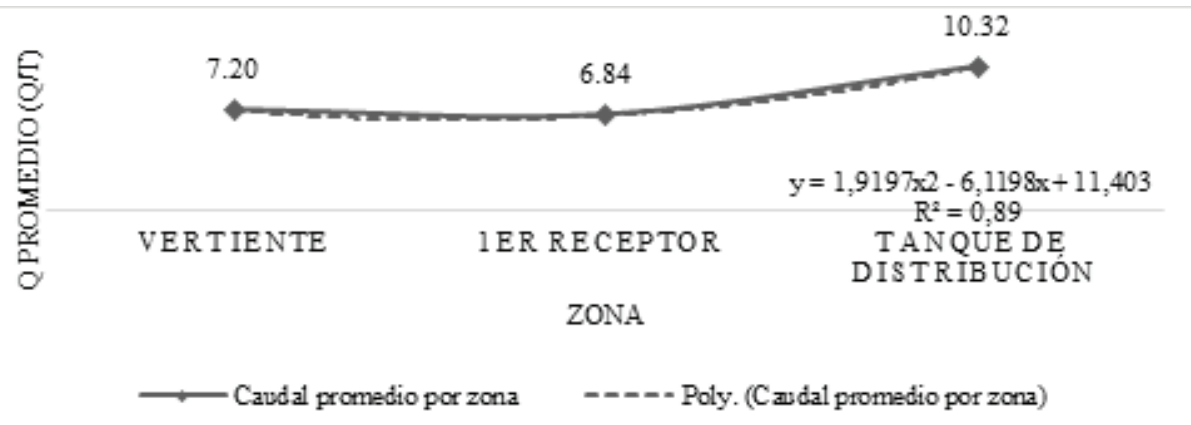

Figure 6

Caudal por zona de aforo.

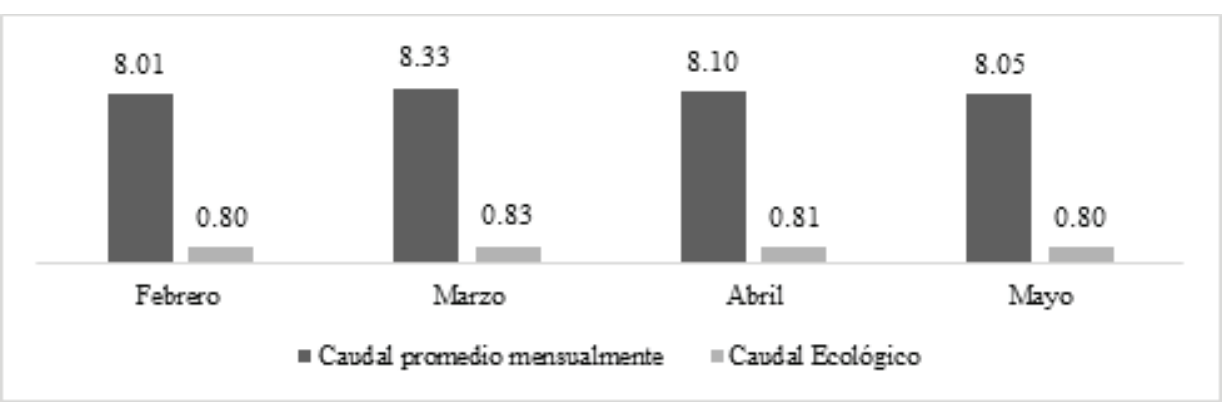

Figure 7

Caudal ecológico mensual correspondiente a los meses de febrero a mayo.

\section{Conclusiones}

Se recolectaron 30 muestras agrupados en 27 especies, 26 géneros, 17 familias y 15.229 individuos, también se recolecto Liquen y Musgo de familia, género y especie no identificados. La especie más representativa en la zona de estudio es $L$. orgiculata con $44,06 \%$ ya que se encontró en 4 de las 5 parcelas de muestreo, además las familias que tuvieron mayor índice de valor de importancia en el área de estudio fueron Rosaceae, Poaceae, Dryopteridaceae Asteraceae y Apiaceae, también se determinó que la parcela con mayor diversidad es la parcela 5 que contiene 11 familias, 12 géneros y 12 especies.

En este estudio se demostró que la diversidad de acuerdo a la equidad (Índice de Shannon) para la parcela 2 es 0,98 y para la parcela 4 es 1,12 interpretándose con diversidad alta, así también estas mismas parcelas de acuerdo a la dominancia (índice de Simpson) se interpreta con diversidad mediana con valores de 0,51 y 0,45, respectivamente, el índice de Sorensen señala que las parcelas de muestreo son disimiles (rango: $0,0-0,35$ ), es decir, las especies solo se encuentran en ciertas zonas de estudio.

El caudal promedio que aporta la zona de estudio páramo del Igualata es de 8,12 L/s, con un caudal ecológico de $0,81 \mathrm{~L} / \mathrm{s}$ situándolo en un caudal 'excelente' que permite satisfacer las necesidades de los pobladores. 
El área de estudio registró una diversidad Florística de 5299 individuos agrupados en 18 familias, 26 géneros y 29 especies, también se recolectó musgo y liquen de familia, género y especie no identificados lo cual suma un total de 31 muestras vegetales.

\section{References}

[1] Mena et al. (2001).

[2] Hofstede R, Segarra P, Mena P. Los Páramos del mundo [The paramos of the world]. Proyecto Atlas Mundial de los Páramos. Quito, Ecuador: Global Peatland Initiative/NC-IUCN/EcoCiencia. 2003. Spanish

[3] Ramón, G. Visiones, usos e intervenciones en los páramos del Ecuador [Visions, uses and interventions in the páramos of Ecuador]. Ecuador: Serie Páramo (Cultura). 2002; (12):43-49. Spanish

[4] Ministerio del Ambiente (MAE). Sistema de clasificación de ecosistemas de Ecuador Continental [Ecosystem classification system of Continental Ecuador,Internet]. 2014 [Recuperado el 19 de Octubre de 2018]. Disponible en: http://app.sni.gob.ec/snilink/sni/PDOT/NIVEL[\%\}2ONACIONAL/MAE/ ECOSISTEMAS/DOCUMENTOS/Sistema.pdf. Spanish

[5] Pauli H, Gottfried M, Lamprecht A, Niessner S, Rumpf S, Winkler M, Steinbauer K. Grabherr G. 2015. Manual para el trabajo de campo del proyecto Gloria. Aproximación al estudio de las cimas Métodos básicos, complementarios y adicionales [Manual for the fieldwork of the Gloria project. Approach to the study of the tops Basic, complementary and additional methods,Internet]. $5^{\text {a }}$ edición. Viena, Austria: GLORIA - Coordinación, Academia Austriaca de Ciencias y Universidad de Recursos Naturales y Ciencias de la Vida. Edición en español a cargo de Benito JL, Villar L, Jaca, España. 2015 [Recuperado el 19 de Octubre de 2018] Disponible en: https://www.researchgate.net/profile/Jose_Luis_Benito_Al onso/publication/282567915_Manual_para_el_trabajo_de_campo_del_proyecto_GLORIA_Aproxima cion_al_estudio_de_las_cimas_Metodos_basico_complementarios_y_adicionales_5_edicion/links/ 5615380308ae4ce3cc6526b3/Manual-para-el-trabajo-de-campo-del-proyecto-GLORIA-Aproximacio n-al-estudio-de-las-cimas-Metodos-basico-complementarios-y-adicionales-5-edicion.pdf. Spanish

[6] Paguay (2018).

[7] Sonco R. Estudio de la diversidad alfa $(\alpha)$ y beta $(\beta)$ en tres localidades de un bosque montano en la región de Madidi, La Paz-Bolivia [Study of alpha $(\alpha)$ and beta $(\beta)$ diversity in three localities of a montane forest in the Madidi region, La Paz-Bolivia,Tesis de Grado. Ingeniero Agrónomo]. La Paz: Universidad Mayor de San Andrés; 2013. Spanish

[8] Aguirre (2006).

[9] Pujos L. Diversidad florística a diferente altitud en el ecosistema páramo de tres comunidades de la organización de segundo grado unión de organizaciones del pueblo Chibuleo [Floristic diversity at different altitudes in the páramo ecosystem of three communities of the second degree organization union of organizations of the Chibuleo people,Tesis de grado. Ingeniero Forestal]. Riobamba: ESPOCH 2013. Spanish

[10] Smith \& Smith (2001).

[11] Valencia C. Índices de diversidad [Diversity indexes,Internet]. 2013 [Recuperado el 19 de Octubre de 2018]. Disponible en: https://es.slideshare.net/ccvalenciac/indices-de-diversidad-ecosistmica. Spanish

[12] Ordoñez et al. (2009).

[13] Bayas D. Diversidad florística a diferente altitud en el ecosistema páramo del cantón Tisaleo provincia de Tungurahua [Floristic diversity at different altitudes in the páramo ecosystem of the Tisaleo canton, Tungurahua province] [Tesis de grado. Ingeniero Forestal]. Riobamba: Escuela Superior Politécnica de Chimborazo ESPOCH; 2015. Spanish

[14] Lozano A, Sánchez J. 2017. Evaluación experimental de una ecuación empírica para la caída de presión en flujo gaseoso [Experimental evaluation of an empirical equation for pressure drop in gas flow,Internet]. 2017 [Recuperado 17 de marzo, del 2018]. Disponible en: http: //repositorio.uncp.edu.pe/bitstream/handle/UNCP/3786/Lozano\{\%\}20Povis\{\%\}20Sanchez\{\%\}20Ochoa. pdf?sequence=1\&isAllowed=y. Spanish

[15] Caranqui J, Lozano P, Reyes J. Composición y diversidad florística de los páramos en la Reserva de Producción de Fauna Chimborazo, Ecuador [Composition and floristic diversity of the páramos in the Chimborazo Fauna Production Reserve, Ecuador]. Quito, Ecuador: Enfoque UTE. 2016 (7):33-42. Spanish

[16] Caranqui (2011).

[17] Hofstede et al. (2013)

[18] Instituto Nacional de Meteorología e Hidrología (INAMHI). Tipos de clima Ecuador [Climate types Ecuador,Internet]. 2017 [Recuperado el 19 de Octubre 2018]. Disponible en: http://www. serviciometeorologico.gob.ec/. Spanish 
[19] Estrella et al. (2015).

[20] Bob R. Cantidad mínima de agua necesaria para uso doméstico [Minimum amount of water required for domestic use,Internet]. 2000 [Recuperado el 19 de Octubre de 2018]. Disponible en: http://www. disasterinfo.net/Agua/pdf/9-UsoDomestico.pdf. Spanish

[21] Bozeman (1976).

[22] Bello M, Pino M. Medición de presión y caudal [Pressure and flow measurement,Internet]. 2000 [Recuperado 5 de mayo del 2018]. Disponible en: http://biblioteca.inia.cl/medios/biblioteca/boletines/ NR25635.pdf. Spanish

[23] Campo A, Duval V. Diversidad y valor de importancia para la conservación de la vegetación natural. Parque Nacional Lihué Calel (Argentina) [. Diversity and value of importance for the conservation of natural vegetation. Lihué Calel National Park (Argentina)]. Madrid, España: L Revista Anales de Geografía de la Universidad Complutense. 2013; (34):32. Spanish

[24] Corredor E, Fonseca J, Páez E. Los servicios ecosistémicos de regulación: tendencias e impacto en el bienestar humano [Regulating ecosystem services: trends and impact on human well-being]. Colombia: Revista de Investigación Agraria y Ambiental. 2012; (3):78-80. Spanish

[25] Díaz S. Biodiversity regulation of ecosystem services, in Hassan R, Scholes R, Ash N (eds.), Ecosystems and hu-human well-being: Current state and trends, (I) Findings of the Condition and Trends Working Group of the Millennium Ecosystem Assessment. Washington, D.C., Estados Unidos: Island Press. 2006

[26] Hofstede R. Effects of burning and grazing on a Colombian paramo ecosystem [Tesis de doctorado]. Ámsterdam, Holanda: Universidad de Ámsterdam. 1995

[27] Hofstede R, Vásconez S, Cerra M. (Ed.) Vivir en los páramos. Percepciones, vulnerabilidades, capacidades y gobernanza ante el cambio climático [Living in the moors. Perceptions, vulnerabilities, capacities and governance in the face of climate change]. Quito, Ecuador: UICN. 2015. Spanish

[28] Jaramillo A. Plan de manejo de los recursos naturales de la Regional San José de Chazo - Santa Fe de Galán [Natural resources management plan of the San José de Chazo - Santa Fe de Galán Regional]. Riobamba, Ecuador: Estrategia de Conservación articulada al proceso de desarrollo local. 2014. Spanish

[29] López J. Caudal ecológico en Ecuador [Ecological flow in Ecuador,Internet]. IAGUA. 2018 [Recuperado el 19 de Octubre de 2018]/ de: https://www.iagua.es/blogs/juan-calles-lopez/ caudal-ecologico-ecuador. Spanish

[30] Mena P, Hofstede R. Los páramos ecuatorianos [The Ecuatorian paramos]. La Paz, Bolivia: Revista Botánica Económica de los Andes Centrales. 2006. p 94 - 95. Spanish

[31] OVACEN. El Páramo [The paramo,Internet]. 2016 [Recuperado el 19 de Octubre de 2018] Disponible en: https://ecosistemas.ovacen.com/bioma/paramo/. Spanish

[32] Peralta J. Determinación del caudal y calidad de agua mediante bioindicadores en los páramos de la comunidad el calvario, ubicado en el cantón Tisaleo, provincia de Tungurahua [Determination of water flow and quality using bioindicators in the moors of the El Calvario community, located in the Tisaleo canton, Tungurahua province,Tesis de grado. Ingeniera Forestal]. Riobamba: ESPOCH; 2019. Spanish 